\section{ORIGINAL RESEARCH}

\author{
V.A. Nagar \\ J.R. Ye \\ W.H. Ng \\ Y.H. Chan \\ F. Hui \\ C.K. Lee \\ C.C.T. Lim
}

\title{
Diffusion-Weighted MR Imaging: Diagnosing Atypical or Malignant Meningiomas and Detecting Tumor Dedifferentiation
}

\begin{abstract}
BACKGROUND AND PURPOSE: Atypical and malignant meningiomas are uncommon tumors with aggressive behavior and higher mortality, morbidity, and recurrence compared with benign tumors. We investigated the utility of diffusion-weighted (DW) MR imaging to differentiate atypical/malignant from benign meningiomas and to detect histologic dedifferentiation to higher tumor grade.
\end{abstract}

\begin{abstract}
MATERIALS AND METHODS: We retrospectively compared conventional and DW MR images (b-value $1000 \mathrm{~s} / \mathrm{mm}^{2}$ ) acquired on a $1.5 \mathrm{~T}$ clinical scanner between 25 atypical/malignant and 23 benign meningiomas. The optimal cutoff for the absolute apparent diffusion coefficient (ADC) and normalized $A D C$ (NADC) ratio to differentiate between the groups was determined by using receiver operating characteristic $(\mathrm{ROC})$ analysis.
\end{abstract}

\begin{abstract}
RESULTS: Irregular tumor margins, peritumoral edema, and adjacent bone destruction occurred significantly more often in atypical/malignant than in benign meningiomas. The mean ADC of atypical/ malignant meningiomas $\left(0.66 \pm 0.13 \times 10^{-3} \mathrm{~mm}^{2} / \mathrm{s}\right)$ was significantly lower compared with benign meningiomas $\left(0.88 \pm 0.08 \times 10^{-3} \mathrm{~mm}^{2} / \mathrm{s} ; P<.0001\right)$. Mean NADC ratio in the atypical/malignant group $(0.91 \pm 0.18)$ was also significantly lower than the benign group $(1.28 \pm 0.11 ; P<.0001)$, without overlap between groups. ROC analysis showed that ADC and NADC thresholds of $0.80 \times 10^{-3}$ $\mathrm{mm}^{2} / \mathrm{s}$ and 0.99 , respectively, had the best accuracy: at the NADC threshold of 0.99 , the sensitivity and specificity were $96 \%$ and $100 \%$, respectively. Two patients had isointense benign tumors on initial DW MR imaging, and these became hyperintense with the decrease in ADC and NADC below these thresholds when they progressed to atypical and malignant meningiomas on recurrence.
\end{abstract}

CONCLUSIONS: ADC and NADC ratios in atypical/malignant meningiomas are significantly lower than in benign tumors. Decrease in ADC and NADC on follow-up imaging may suggest dedifferentiation to higher tumor grade.

$\mathbf{M}$ eningiomas comprise $14 \%-20 \%$ of all intracranial tumors, with a higher incidence of up to $35.2 \%$ among Asians and Africans. ${ }^{1}$ Although they are generally benign tumors, up to $10 \%$ of meningiomas are atypical or malignant, characterized by nuclear disorganization, necrosis, prominent nucleoli, and increased mitoses on histology. Because of their aggressive behavior, atypical/malignant meningiomas are associated with high morbidity and mortality and may invade the adjacent bone and brain parenchyma. They are also more prone to recur in $29 \%-41 \%$ of patients than typical meningiomas, where the recurrence rate is between $7 \%-20 \%$. $^{2} \mathrm{Al}-$ though typical extra-axial benign meningiomas are easily diagnosed, distinction from more malignant histologic grades by CT or conventional MR imaging is difficult. ${ }^{3}$ Neuroimaging features, such as heterogeneous appearance, heterogeneous enhancement, marked perilesional edema, irregular cerebral surface, mushrooming on the outer edge of the lesion, and bone destruction, are not unique or reliable for diagnos-

Received October 3, 2007; accepted after revision December 21.

From the Departments of Neuroradiology (V.A.N., J.R.Y., F.H., C.C.T.L.) and Neurosurgery (W.H.N., C.K.L.), National Neuroscience Institute, Singapore; Biostatistics (Y.H.C.) and Diagnostic Radiology (C.C.T.L.), Yong Loo Lin School of Medicine, National University of Singapore, Singapore.

This study was partly supported by the National Healthcare Group and Singapore Health Service grants PTD/03004 and PTD/01010.

Please address correspondence to C.C. Tchoyoson Lim, Department of Neuroradiology, National Neuroscience Institute, 11 Jalan Tan Tock Seng, Singapore 308433; e-mail: tchoyoson_lim@nni.com.sg

三E indicates article with supplemental on-line tables.

DOI 10.3174/ajnr.A0996 ing atypical/malignant meningiomas. ${ }^{4-8}$ A diagnostic method that can differentiate between benign and atypical/ malignant meningiomas would, therefore, be desirable for surgical planning.

Diffusion-weighted (DW) MR imaging has been used to study primary brain tumors, including histologic grading of gliomas and response to treatment. ${ }^{9-16}$ Only a few studies have evaluated DW MR imaging for grading meningiomas, and although some have found that apparent diffusion coefficient (ADC) of atypical/malignant meningiomas was significantly lower than benign meningiomas, ${ }^{2,17}$ others have not duplicated these results. ${ }^{16}$ Furthermore, the accuracy and threshold $\mathrm{ADC}$ to distinguish between benign and atypical/malignant meningiomas has not been established. There is also a paucity of literature on the DW MR imaging appearance of malignant transformation of benign meningiomas to higher grade tumors. In this study, we compared DW MR imaging between benign and atypical/malignant groups of meningiomas to estimate the cutoff ADC value for optimal tumor grading and describe imaging features of dedifferentiation of benign meningiomas.

\section{Materials and Methods}

\section{Patient Selection}

We retrospectively reviewed the neuroradiology imaging data base from January 1999 to December 2006 at a tertiary referral neurologic hospital that performs 150 brain tumor surgical procedures per annum. ${ }^{18}$ Twenty-five consecutive atypical and malignant meningiomas proved by histologic examination (representing 6.9\% of all me- 

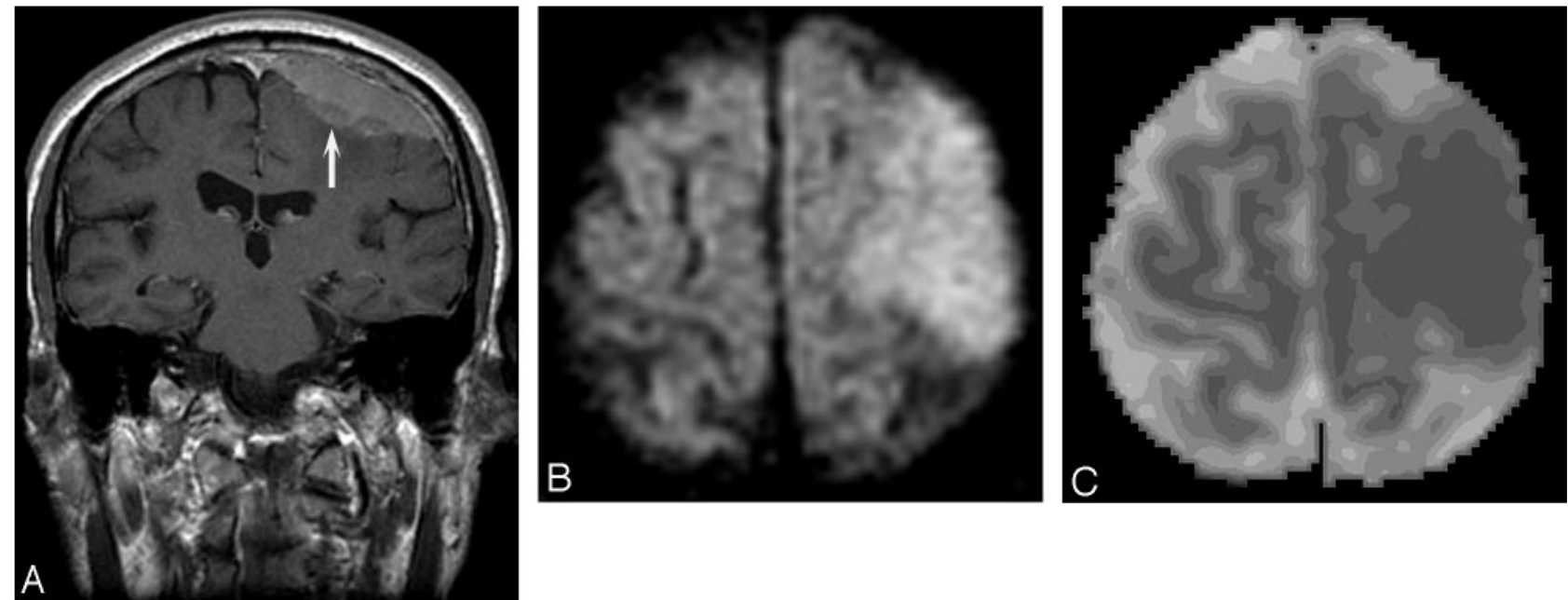

Fig 1. Patient 34, atypical meningioma in a 73-year-old man. $A$, Enhanced coronal T1 image shows an en plaque meningioma (arrow) over the left frontoparietal region, with ill-defined margins. $B$, DW MR image demonstrates the mass to be hyperintense. C, ADC map shows decreased signal intensity compared with normal white matter (absolute $A D C=0.63 \times 10^{-3}$ $\mathrm{mm}^{2} / \mathrm{s} ; \mathrm{NADC}$ ratio $=0.88$.
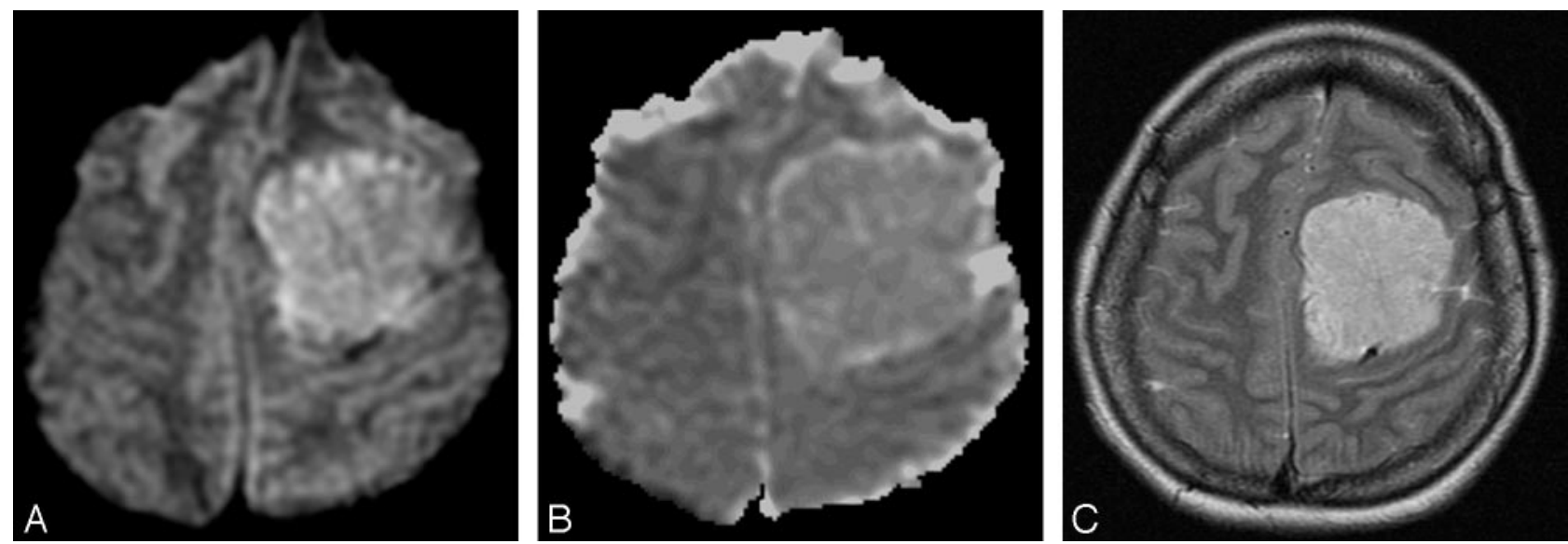

Fig 2. Patient 18 , benign meningioma in a 37-year-old woman. $A$, DW MR image showing the tumor in the left frontal region to be hyperintense. $B$, ADC map showing increased signal intensity compared with normal white matter (absolute $\mathrm{ADC}=0.98 \times 10^{-3} \mathrm{~mm}^{2} / \mathrm{s}$; NADC ratio $=1.46$ ). C, Axial T2-weighted image shows that the hyperintensity in $A$ was probably due to $T 2$ shinethrough effect.

ningiomas) and studied by using presurgical DW MR imaging were selected. Atypical and malignant meningiomas were diagnosed based on the World Health Organization (WHO) classification of grades II and III tumors, respectively. A control group of 23 patients with benign meningiomas was also selected from the benign meningothelial meningioma subtype from the same data base. There is evidence that ADC values of meningothelial meningioma may be the lowest among the different subtypes of benign meningiomas ${ }^{2}$ and, hence, may represent a suitable homogenous control group for comparison with the low ADC in atypical/malignant tumors. Patients who did not have DW MR imaging or whose studies were technically unsuccessful were excluded from the study. The study was approved by the institutional review board as part of ongoing studies in brain tumors and the neuroradiology data base.

\section{MR Protocol}

All of the patients underwent MR imaging study on a $1.5 \mathrm{~T}$ clinical scanner (GE Medical Systems, Milwaukee, Wis) using standard head coil with $240 \times 240$-mm FOV according to the tumor protocol in our hospital within a week before surgical resection. Conventional MR images consisted of at least axial fast spin-echo T2-weighted images
(TR/TE/3000-5400/100-105 msec) and contrast-enhanced images T1-weighted images (TR/TE $=400-660 / 12-20 \mathrm{~ms})$ after intravenous contrast injection (gadopentetate dimeglumine $=0.1 \mathrm{mmol} / \mathrm{kg}$ ). $\mathrm{DW}$ MR imaging was acquired in the axial plane by using a single-shot, spin-echo echo-planar imaging sequence $(\mathrm{TR} / \mathrm{TE}=10,000 / 68.4 \mathrm{~ms})$ with b-values of 0 and $1000 \mathrm{~s} / \mathrm{mm}^{2}$ in 3 orthogonal directions.

\section{Data Calculation and Analysis}

Two investigators (V.A.N. and C.C.T.L.) evaluated the MR images by consensus reading. Conventional MR images were analyzed for the maximum tumor diameter, presence of perilesional edema, irregular tumor edge, inhomogeneous enhancement characteristics, and bone destruction. Isotropic (trace, ie, the summation of 3 orthogonal directions) DW images were visually inspected and classified as hyperintense, isointense, or hypointense compared with normal white matter. The intratumoral ADC values were measured using the manufacturer's software (FuncTool; GE Medical Systems). To minimize variability, the regions of interest (ROIs), varying from 30 to $130 \mathrm{~mm}^{2}$, were placed manually in the solid part of the tumor, avoiding any cystic or calcified areas. In cases of very large tumors, the mean value of 3 ROIs was recorded. 

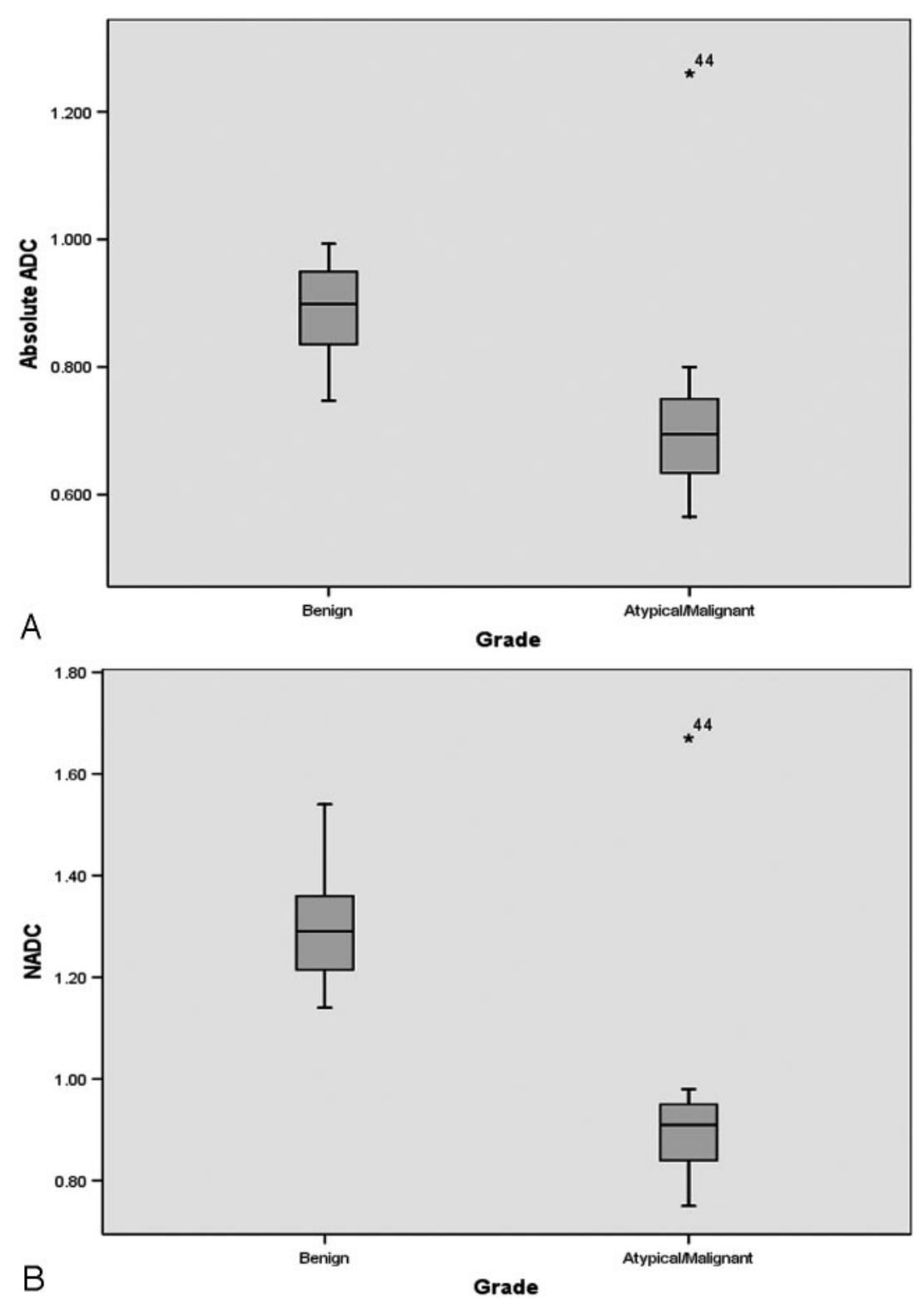

Fig 3. Box and whisker plot comparing $A D C$ values between groups. A, Absolute intratumoral $A D C$ values in benign and atypical/malignant meningiomas showing some overlap between groups. A single outlier (patient 44) in the atypical/ malignant group had a recurrent malignant meningioma of the rhabdoid variant. $B$, There is no overlap in NADC ratios between benign and atypical/malignant meningiomas.

\section{Results}

The results of analysis of MR images in benign and atypical/malignant meningiomas are summarized in supplemental on-line Tables 1 and 2. Twenty-three patients had benign meningothelial meningiomas; 2 of these patients (patients 12 and 13) had dedifferentiation to higher grade tumors on recurrence after 1 and 4 years, respectively. Twenty-one other patients had a final diagnosis of atypical/malignant meningioma; 2 of these patients with atypical meningioma (patients 37 and 41) also had tumor recurrence and dedifferentiation to malignant meningioma at subsequent surgery after 1 year. A total of 25 atypical/malignant meningiomas were studied, including the 2 patients with dedifferentiation from benign tumors.

On conventional MR imaging, the mean maximum diameter of atypical/malignant meningiomas $(5.6 \pm 2.5 \mathrm{~cm})$ was not significantly larger than that of benign meningiomas $(5.4 \pm 1.5 \mathrm{~cm} ; P=.72, t$ test). Heterogeneous enhancement pattern was also not significantly different between groups ( $P=.179$, Mann-Whitney $U$ test $)$. Adjacent bone destruction was found in $32 \%$ of atypical/malignant meningiomas but not in benign tumors $(P=.003$, Mann-Whitney $U$ test). Irregular tumor margins $(P=$ .008 , Mann-Whitney $U$ test) and peritu-

Control ADC values were obtained from normal-appearing white matter on the contralateral normal brain tissue unaffected by tumor. The normalized ADC (NADC) ratios were calculated using the formula $\mathrm{NADC}=\mathrm{ADC}$ of the tumor/ADC of the normal white matter, with NADC lower than 1.00 representing relatively restricted diffusion.

All of the analyses were performed using SPSS version 14.0 for Windows (SPSS, Chicago, Ill). The differences in mean tumor diameter, mean ADC, and mean NADC ratio between the benign and atypical/malignant groups of meningioma were compared using the 2-sample $t$ test when normality and homogeneity assumptions were satisfied, otherwise the equivalent nonparametric Mann-Whitney $U$ test was applied. Logistic regression analysis was performed to assess the predictive value of conventional MR imaging features for distinguishing groups of meningiomas. Statistical significance was set at $P<.05$. The optimal cutoff for the above significant variables to differentiate between benign and atypical/malignant meningioma groups was determined using receiver operating characteristic (ROC) curve analysis, with sensitivity, specificity, and positive and negative predictive values presented. moral edema ( $P=.012$, Mann-Whitney $U$ test $)$ were also features seen significantly more often in atypical/malignant meningiomas. Logistic regression analysis, however, showed that these features were not statistically significant to correctly predict the tumor grades of meningiomas. The overall accuracy of conventional MR imaging to predict patients having atypical/ malignant meningioma was $72.3 \%$, with a sensitivity of $64.0 \%$, specificity of $81.8 \%$, positive predictive value (PPV) of $80.0 \%$, and negative predictive value (NPV) of $66.7 \%$.

On DW MR images, the signal intensity of 23 benign meningiomas was variable, with 4 hypointense, 9 isointense, and 10 hyperintense benign tumors. Of 25 atypical/malignant meningiomas, 2 were hypointense, 3 were isointense, 19 were hyperintense (Fig 1), and 1 had mixed hypointense and isointense signal intensity. ADC maps showed that all of the benign meningiomas were hyperintense or isointense compared with normal white matter (Fig 2). On the other hand, ADC maps of atypical/malignant meningiomas showed isointensity or hypointensity, except patient 44, whose tumor was hyperintense and isointense. Absolute ADC in benign meningiomas ranged 

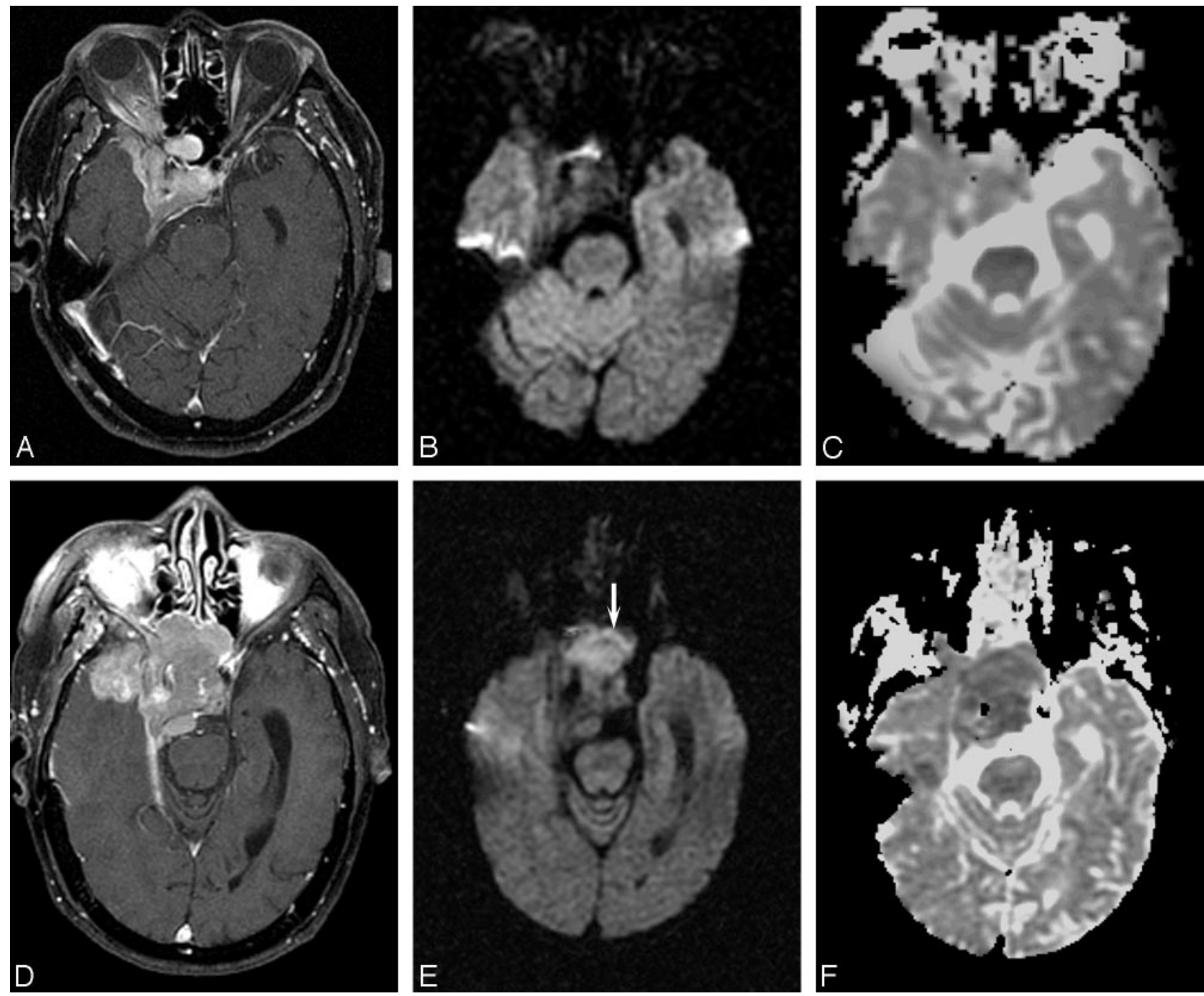

Fig 4. Patient 12, right sphenoid wing meningioma in a 79-year-old man. $A$, Before first surgery, contrast-enhanced axial $\mathrm{T} 1$ image shows an extra-axial enhancing mass lesion at the right sphenoid wing causing cavernous sinus obstruction. $B$, Corresponding DW MR image demonstrates the mass to be isointense. $C, A D C$ map shows isointensity labsolute $A D C=0.90 \times$ $10^{-3} \mathrm{~mm}^{2} / \mathrm{s}$; NADC ratio $=1.23$ ). Histologic examination showed benign meningothelial meningioma. $D$, Follow-up contrast-enhanced axial T1-weighted image before second resection shows the recurrent mass to be more extensive. E, Corresponding DW MR image of the tumor before second resection (arrow) is now hyperintense. F, ADC map shows hypointensity (absolute ADC has decreased to $0.60 \times 10^{-3} \mathrm{~mm}^{2} / \mathrm{s}$, and NADC ratio decreased to 0.77 ). Histologic examination revealed dedifferentiation to atypical meningioma.

from 0.75 to $0.99 \times 10^{-3} \mathrm{~mm}^{2} / \mathrm{s}$, whereas the NADC ratio ranged from 1.14 to 1.54 . In atypical/malignant meningiomas, absolute ADC ranged from 0.58 to $1.26 \times 10^{-3} \mathrm{~mm}^{2} / \mathrm{s}$, and NADC ranged from 0.80 to 1.67 .

All of the atypical/malignant meningiomas had absolute ADC and NADC ratios less than 1.00 with the exception of a single patient with recurrent malignant rhabdoid meningioma (patient 44). The mean and SD of absolute intratumoral ADC in benign meningiomas was $0.88 \pm 0.08 \times 10^{-3} \mathrm{~mm}^{2} / \mathrm{s}$, and in atypical/malignant meningiomas the mean $\mathrm{ADC}$ of $0.66 \pm$ $0.13 \times 10^{-3} \mathrm{~mm}^{2} / \mathrm{s}$ was significantly lower $(P<.0001)$. The mean NADC ratio in the atypical/malignant group $(0.91 \pm$ 0.18 ) was also significantly lower than in the benign group $(1.28 \pm 0.11 ; P<.0001)$. Although absolute ADC values showed considerable overlap, there was no NADC ratio overlap between groups (Fig 3). There was no significant difference in the mean ADC and NADC ratio between atypical and malignant meningiomas ( $P=.780$ and 0.999 , respectively). Using the ROC curves, the optimal cutoff for absolute ADC was
$0.80 \times 10^{-3} \mathrm{~mm}^{2} / \mathrm{s}$ with sensitivity of $96 \%$, specificity of $82.6 \%$, PPV of $85.7 \%$, and NPV of $95 \%$. ROC analysis showed that the NADC ratio of 0.99 had the highest accuracy, with sensitivity of $96 \%$, specificity of $100 \%$, PPV of $100 \%$ and NPV of $95.8 \%$.

There were 4 patients in our series who had undergone DW MR imaging studies before both surgical episodes with tumors that underwent anaplastic dedifferentiation to higher grade. Two patients (Patient 12 and 13) progressed from benign to atypical and malignant meningioma, respectively, on recurrence. In both cases, the initial benign tumor was isointense on DW MR imaging, becoming hyperintense on subsequent DW MR image when they dedifferentiated (Fig 4). This change in DW MR image signal intensity was accompanied by a decrease in the $\mathrm{ADC}$ values from $0.88 \times 10^{-3} \mathrm{~mm}^{2} / \mathrm{s}$ to $0.60 \times 10^{-3} \mathrm{~mm}^{2} / \mathrm{s}$ and from $0.90 \times 10^{-3}$ $\mathrm{mm}^{2} / \mathrm{s}$ to $0.63 \times 10^{-3} \mathrm{~mm}^{2} / \mathrm{s}$ in both patients crossing below the ROC threshold of $0.80 \times 10^{-3} \mathrm{~mm}^{2} / \mathrm{s}$. The NADC ratio was also reduced from 1.23 to 0.77 and 1.23 to 0.85 , respectively, again decreasing below the threshold level of 0.99 . 
Two other patients (patients 37 and 41) with atypical meningiomas had tumor recurrence and dedifferentiated to malignant meningiomas on subsequent histologic examination. In both patients, the atypical and malignant meningiomas were similar on conventional MR imaging appearance and were also hyperintense on DW MR images. After dedifferentiation, the absolute ADC decreased in both patients from $0.65 \times 10^{-3} \mathrm{~mm}^{2} / \mathrm{s}$ to $0.56 \times 10^{-3} \mathrm{~mm}^{2} / \mathrm{s}$ and $0.67 \times 10^{-3}$ $\mathrm{mm}^{2} / \mathrm{s}$ to $0.59 \times 10^{-3} \mathrm{~mm}^{2} / \mathrm{s}$, respectively. The NADC ratios were unchanged in patient 37 (0.78 in both atypical and malignant meningiomas) and decreased in patient 41 (from 0.84 to 0.75$)$. The ADC and NADC values in all instances were below the thresholds consistent with atypical/malignant meningioma.

\section{Discussion}

We found that the mean ADC value and NADC ratio were lower (ie, relatively restricted diffusion) in atypical/malignant meningiomas than in benign tumors. Although some MR imaging features, such as ill-defined tumor margins, adjacent bone destruction, and peripheral edema, ${ }^{19}$ were suggestive of atypical/malignant meningiomas, logistic regression analysis of conventional MR imaging features did not show significant correlation with the histologic grade. These findings were consistent with previous CT and MR imaging reports, which found that none of these features were unique and could also be seen in benign meningiomas. ${ }^{4-8}$ Two previous studies have investigated using DW MR imaging to grade meningiomas. ${ }^{2,17}$ These studies, involving a total of 9 atypical and 2 malignant meningiomas, showed that the mean ADC values in atypical/ malignant tumors were significantly lower compared with benign meningioma. ${ }^{2,17}$ Our results in a larger patient population are consistent with these reports. Various theories have been proposed to explain the decreased ADC in high-grade tumors, including increased tumor cellularity, tumor matrices, fibrous or gliotic tissues, or a combination of these factors. ${ }^{10}$ Water diffusion in biologic tissue is highly dependent on the ratio of intracellular to extracellular space, and increased cellularity in high-grade tumors may decrease the fraction of extracellular space, thus restricting net water diffusion. ${ }^{20,21}$ Furthermore, compared with benign meningiomas, atypical and malignant meningiomas have increased mitotic activity, necrosis, prominent nuclei, and small cells with an increased amount of intracellular complex protein molecules and high nucleus-to-cytoplasm ratio, contributing to decreased free diffusion of water. ${ }^{22}$

Using ROC curves, we found that the optimal ADC and NADC thresholds that could differentiate atypical/malignant meningiomas were $0.80 \times 10^{-3} \mathrm{~mm}^{2} / \mathrm{s}$ and 0.99 , respectively. Using these thresholds, ADC correctly predicted $96 \%$ of atyp$\mathrm{ical} /$ malignant and $83 \%$ of benign meningiomas, and NADC correctly predicted $96 \%$ of atypical/malignant and all of the benign meningiomas. This suggests that NADC ratios might be more specific and accurate than absolute ADC to preoperatively distinguish between high-grade and low-grade meningiomas. The measurement of absolute ADC values may vary across different scanners, and the NADC ratio may be a less variable parameter to use. The NADC ratio minimizes the differences in absolute ADC values that may be obtained with different DW imaging sequences and hardware configurations, thereby eliminating interimage variability. Furthermore, normalization of ADC by obtaining ratios compared with contralateral normal values has been used in differentiating and grading of intra-axial tumors and may be preferable to absolute ADC values. ${ }^{15,23}$ Our statistical analysis was limited by a single outlier (patient 44). This patient had a rhabdoid variant (WHO grade 3 ) of malignant meningioma, which had irregular margins and peritumoral edema but atypically high ADC and NADC values that were more characteristic of benign meningiomas and would, thus, have been misclassified as benign using our ROC criteria. Rhabdoid variants are biologically and clinically aggressive with sheets of loosely cohesive cells with eccentric nuclei and hyaline, paranuclear inclusions. ${ }^{24}$ They tend to arise from meningiomas with more benign and typical morphology $y^{24,25}$ and may, therefore, exhibit the histopathologic features more characteristic of benign meningiomas rather than the restriction in diffusion more typical of the other subtypes of atypical/malignant meningiomas. However, in our single case, we were unable to postulate the reason for the tumor to have increased water diffusion compared with other malignant meningiomas. In a previous study, there has been some suggestion that different subtypes of benign meningiomas may have different ranges of ADC values, ${ }^{2}$ but larger studies would be necessary to verify these findings and to determine whether there are distinctive features in rhabdoid variants that might preclude the use of DW MR imaging for grading these cases.

Although from our ROC analysis, ADC and NADC ratios could accurately distinguish benign from atypical/malignant meningioma, the signal intensity of tumors on DW MR imaging was more variable; not all of the high-grade tumors were hyperintense, and some benign tumors were also hyperintense. This hyperintensity is probably due to the contribution of T2 and spin attenuation on the trace DW MR image, often referred to as the "T2 shinethrough" effect. ${ }^{26,27}$ Hence, relative restriction of the $\mathrm{ADC}$ values was more useful than increased signal intensity in distinguishing tumor groups. However, the ADC or NADC ratios between the atypical and malignant groups were not significantly different. Our study was also limited by the small number of patients who had follow-up imaging and the lack of serial DW MR imaging in patients with recurrent benign tumor compared with recurrent atypical/malignant meningiomas. Furthermore, only the meningothelial subtype of benign meningioma was included in our study, and other subtypes were not evaluated. There is some evidence that the ADC values of meningothelial meningioma may be the lowest among the different subtypes of benign meningiomas, ${ }^{2}$ and, hence, they may represent a suitable homogenous control group for comparison with the low ADC in atypi$\mathrm{cal} /$ malignant tumors. However, our results are preliminary, and the ADC and NADC thresholds may be affected by scanner and operator factors and should not be considered "hard and fast" rules. Prospective studies of larger patient populations with interobserver measurements would be helpful for validation of these results. Diffusion tensor imaging, involving 6 or more gradient directions, can also be used to provide information about the tumor consistency and to potentially study the benign and malignant subtypes of meningioma. ${ }^{28,29}$

Two patients with benign meningiomas had recurrence of atypical/malignant tumors on follow-up imaging, which showed 
a change from isointensity to hyperintensity on DW MR imaging and a reduction in the ADC value and NADC ratio below the threshold for higher tumor grade. Although, as a group, the qualitative analysis of the signal intensity on DW MR images was variable and could not be used for distinguishing groups, in these 2 patients, tumor dedifferentiation could be detected visually on presurgical neuroimaging. A method to predict tumor progression and histologic dedifferentiation on serial imaging during surveillance of postsurgical patients would be desirable. ${ }^{30}$ Early intervention and aggressive adjuvant therapy, such as fractionated radiation therapy, may be helpful to improve outcome and increase overall survival rates. ${ }^{31}$ Several methods, including positron-emission tomography, perfusion MR imaging, and MR spectroscopy, have been proposed to study tumor grading and malignant progression in glial neoplasms. ${ }^{32-37}$ Spectroscopic methods in particular may be useful in eliciting the metabolic correlates of genetic progression of gliomas or the malignant potential of meningiomas ${ }^{38,39}$ during follow-up neuro-oncologic imaging. The role of decreasing ADC as a biomarker of worsening histologic grade has been less widely studied in intracranial neoplasms ${ }^{40-42}$ (M.S. Xu and C.C.T. Lim, unpublished data), and to the best of our knowledge, there have been no previous studies evaluating DW MR imaging in dedifferentiation of meningiomas.

In this study, we found that DW MR imaging can distinguish benign from atypical/malignant meningiomas; NADC may be a more accurate predictor of tumor grade than absolute ADC. During follow-up imaging for tumor recurrence, a decrease in ADC and NADC may suggest dedifferentiation into higher tumor grade. DW MR imaging may be a useful bioimaging marker to study meningioma grading and progression.

\section{References}

1. Das A, Tang WY, Smith DR. Meningiomas in Singapore: demographic and biological characteristics. J Neurooncol 2000;47:153-60

2. Hakyemez B, Yildirim N, Gokalp G, et al. The contribution of diffusionweighted MR imaging to distinguishing typical from atypical meningiomas. Neuroradiology 2006;48:513-20

3. Carpeggiani P, Crisi G, Trevisan C. MRI of intracranial meningiomas: correlations with histology and physical consistency. Neuroradiology 1993;35:532-36

4. Jaaskelainen J, Haltia M, Laasonen E, et al. The growth rate of intracranial meningiomas and its relation to histology: an analysis of 43 patients. Surg Neurol 1985;24:165-72

5. Mahmood A, Caccamo DV, Tomecek FJ, et al. Atypical and malignant meningiomas: a clinicopathological review. Neurosurgery 1993;33:955-63

6. Verheggen R, Finkenstaedt M, Bockermann V, et al. Atypical and malignant meningiomas: evaluation of different radiological criteria based on CT and MRI. Acta Neurochir 1996;65(suppl):66-69

7. Zee CS, Chin T, Segall HD, et al. Magnetic resonance imaging of meningiomas. Semin Ultrasound CT MR 1992;13:154-69

8. Modha A, Gutin PH. Diagnosis and treatment of atypical and anaplastic meningiomas: a review. Neurosurgery 2005;57:538-50

9. Krabbe K, Gideon P, Wagn P, et al. MR diffusion imaging of human intracranial tumours. Neuroradiology 1997;39:483-89

10. Sugahara T, Korogi Y, Kochi M, et al. Usefulness of diffusion-weighted MRI with echo-planar technique in the evaluation of cellularity in gliomas. J Magn Reson Imaging 1999;9:53-60

11. Gupta R, Sinha U, Cloughesy T, et al. Inverse correlation between choline magnetic resonance spectroscopy signal intensity and the apparent diffusion coefficient in human glioma. Magn Reson Med 1999;41:2-7

12. Gupta R, Cloughesy T, Sinha U, et al. Relationships between choline magnetic resonance spectroscopy, apparent diffusion coefficient and quantitative histopathology in human glioma. Neuro-oncology 2000;50:215-26

13. Stadnik TW, Chaskis C, Michotte A, et al. Diffusion-weighted MR imaging of intracerebral masses: comparison with conventional MR imaging and histologic findings. AJNR Am J Neuroradiol 2001;22:969-76

14. Kono $\mathrm{K}$, Inoue $\mathrm{Y}, \mathrm{Nakayama} \mathrm{K}$, et al. The role of diffusion-weighted imaging in patients with brain tumors. AJNR Am J Neuroradiology 2001;22:1081-88
15. Bulakbasi N, Guvenc I, Onguru O, et al. The added value of the apparent diffusion coefficient calculation to magnetic resonance imaging in the differentiation and grading of malignant brain tumors. J Comput Assist Tomogr 2004;28:735-46

16. Yamasaki F, Kurisu K, Satoh K, et al. Apparent diffusion coefficient of human brain tumors at MR imaging. Radiology 2005;235:985-91

17. Filippi CG, Edgar MA, Ulug AM, et al. Appearance of meningiomas on diffusion-weighted images: correlating diffusion constants with histopathologic findings. AJNR Am J Neuroradiol 2001;22:65-72

18. Yang GL, Tan YF, Loh SC, et al. Neuroradiology imaging database: using picture archive and communication systems for brain tumour research. Sing Med J 2007;48:342-46

19. Tan WL, Wong JHY, Liew D, et al. Aquaporin-4 is correlated with peritumoural oedema in meningiomas. Ann Acad Med Singapore 2004;33:S87-89

20. Vorisek I, Hajek M, Tintera J, et al. Water ADC, extracellular space volume, and tortuosity in the rat cortex after traumatic injury. Magn Reson Med 2002;48:994-1003

21. Szafer A, Zhong J, Anderson AW, et al. Diffusion-weighted imaging in tissues: theoretical models. NMR Biomed 1995;8:289-96

22. Buetow MP, Buetow PC, Smirniotopoulos JG. Typical, atypical, and misleading features in meningioma. Radiographics 1991;11:1087-106

23. Hein PA, Eskey CJ, Dunn JF, et al. Diffusion-weighted imaging in the follow-up of treated high-grade gliomas: tumor recurrence versus radiation injury. AJNR Am J Neuroradiol 2004;25:201-09

24. Kepes JJ, Moral LA, Wilkinson SB, et al. Rhabdoid transformation of tumor cells in meningiomas: a histologic indication of increased proliferative activity: report of four cases. Am J Surg Pathol 1998;22:231-38

25. Perry A, Scheithauer BW, Stafford SL, et al. "Rhabdoid" meningioma: an aggressive variant. Am J Surg Pathol 1998;22:1482-90

26. Provenzale JM, Engelter ST, Petrella JR, et al. Use of MR exponential diffusionweighted images to eradicate T2 shine-through phenomena on diffusionweighted MR images. AJR Am J Roentgenol 1999;172:537-39

27. Burdette JH, Elster AD, Ricci PE. Acute cerebral infarction: quantification of spin-density and T2 shine-through phenomena on diffusion-weighted MR images. Radiology 1999;212:333-39

28. Tropine A, Dellani PD, Glaser M, et al. Differentiation of fibroblastic meningiomas from other benign subtypes using diffusion tensor imaging. J Magn Reson Imaging 2007;25:703-08

29. Kashimura H, Inoue T, Ogasawara K, et al. Prediction of meningioma consistency using fractional anisotropy value measured by magnetic resonance imaging. J Neurosurg 2007;107:784-87

30. Jeuken WM, Sprenger HE, Vermeer H, et al. Chromosomal imbalances in primary oligodendroglial tumors and their recurrences: clues about malignant progression detected using comparative genomic hybridization. J Neurosurg 2002;96:559-64

31. Milosevic MF, Frost PJ, Laperriere NJ, et al. Radiotherapy for atypical or malignant intracranial meningioma. Int J Radiat Oncol Biol Phys 1996;34:817-22

32. Fulham MJ, Bizzi A, Dietz MJ, et al. Mapping of brain tumor metabolites with proton MR spectroscopic imaging: Clinical relevance. Radiology 1992;185:679-86

33. Tedeschi G, Lundbom N, Raman R, et al. Increased choline signal coinciding with malignant degeneration of cerebral gliomas: a serial proton magnetic resonance spectroscopy imaging study. J Neurosurg 1997;87:516-24

34. Fuss M, Wenz F, Essig M, et al. Tumor angiogenesis of low-grade astrocytomas measured by dynamic susceptibility contrast-enhanced MRI (DSC-MRI) is predictive of local tumor control after radiation therapy. Int J Radiation Oncology Biol Phys 2001;51:2:478-82

35. Law M, Oh S, Babb J, et al. Low-grade gliomas: dynamic susceptibilityweighted contrast-enhanced perfusion MR imaging-Prediction of patient clinical response. Radiology 2006;238:658-67

36. Floeth FW, Pauleit D, Sabel M, et al. Prognostic value of O-(2-18F-fluoroethyl)-Ltyrosine PET and MRI in low-grade glioma. $\mathrm{J} \mathrm{Nucl} \mathrm{Med}$ 2007;48:519-27

37. Xu M, See SJ, Ng WH, et al. Comparison of magnetic resonance spectroscopy and perfusion weighted imaging in pre-surgical grading of oligodendroglial tumors. Neurosurgery 2005;56:919-26

38. Shino A, Nakasu S, Matsuda M, et al. Noninvasive evaluation of the malignant potential of intracranial meningiomas performed using proton magnetic resonance spectroscopy. J Neurosurg 1999;91:928-34

39. Lehnhardt F-G, Bock C, Rohn G, et al. Metabolic differences between primary and recurrent human brain tumors: a ${ }^{1} \mathrm{H}$ NMR spectroscopic investigation. NMR Biomed 2005; 18:371-82

40. Yin $H$, Parmar $H$, Yeo $T$, et al. Malignant transformation of oligendendroglioma: MR spectroscopy and diffusion-weighed MR imaging features. Eur J Radiol Extra 2004;49:1-6

41. Inaoka T, Takahashi K, Miyokawa N, et al. Solitary fibrous tumor of the pleura: apparent diffusion coefficient (ADC) value and ADC map to predict malignant transformation. J Magn Reson Imaging 2007;26:155-58

42. Schubert MI, Wilke M, Muller-Weihrich S, et al. Diffusion-weighted magnetic resonance imaging of treatment-associated changes in recurrent and residual medulloblastoma: preliminary observations in three children. Acta Radiol 2006;47:1100-04 